\title{
12 Wieviel Text enthalten die noch geschlossenen Rollen? Ein Quantifizierungsversuch
}

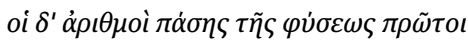

Pythagoreer (nach Aristoteles)

Wieviel Text verbirgt sich noch in den ungeöffneten Rollen der Officina dei papi$r i$ in Neapel? Diese angesichts der „absehbaren“ virtuellen Öffnung dieser Rollen eigentlich naheliegende Frage wurde bisher noch nicht gestellt, d.h. noch kein nicht-trivialer Quantifizierungsversuch gewagt. Jedoch kann eine quantitative Orientierung mit den neu entwickelten mathematisch-bibliometrischen Methoden (siehe 13.1.4) ohne allzu viel Aufwand und mit einer gewissen Aussagekraft gewonnen werden (Wie viele Kolumnen? Wie viele Wörter? Wie viele Referenzseiten?).

$\mathrm{Zu}$ diesem Zweck sollten wir uns vor Augen führen, dass die verschiedenen Wicklungen einer Papyrusrolle (genauer: des Querschnitts einer Papyrusrolle), welche genaugenommen einer archimedischen Spirale gleicht (siehe 13.1.4), approximativ als konzentrische Kreise um den Mittelpunkt der Rolle herum angesehen werden können. Sie sind je nach „Festigkeit“ der Wicklung bzw. Dicke des Papyrus zwischen 0,1 und 0,25 mm voneinander entfernt. ${ }^{148}$ Daraus folgt, dass die Breite der Wicklungen im aufgewickelten Zustand der Papyri in der Regel um etwa 1-2 mm pro Wicklung abnimmt (von links nach rechts).

148 Dazu Holger Essler, „Rekonstruktion von Papyrusrollen auf mathematischer Grundlage,“ CErc 38 (2008): (273-307), 286. 

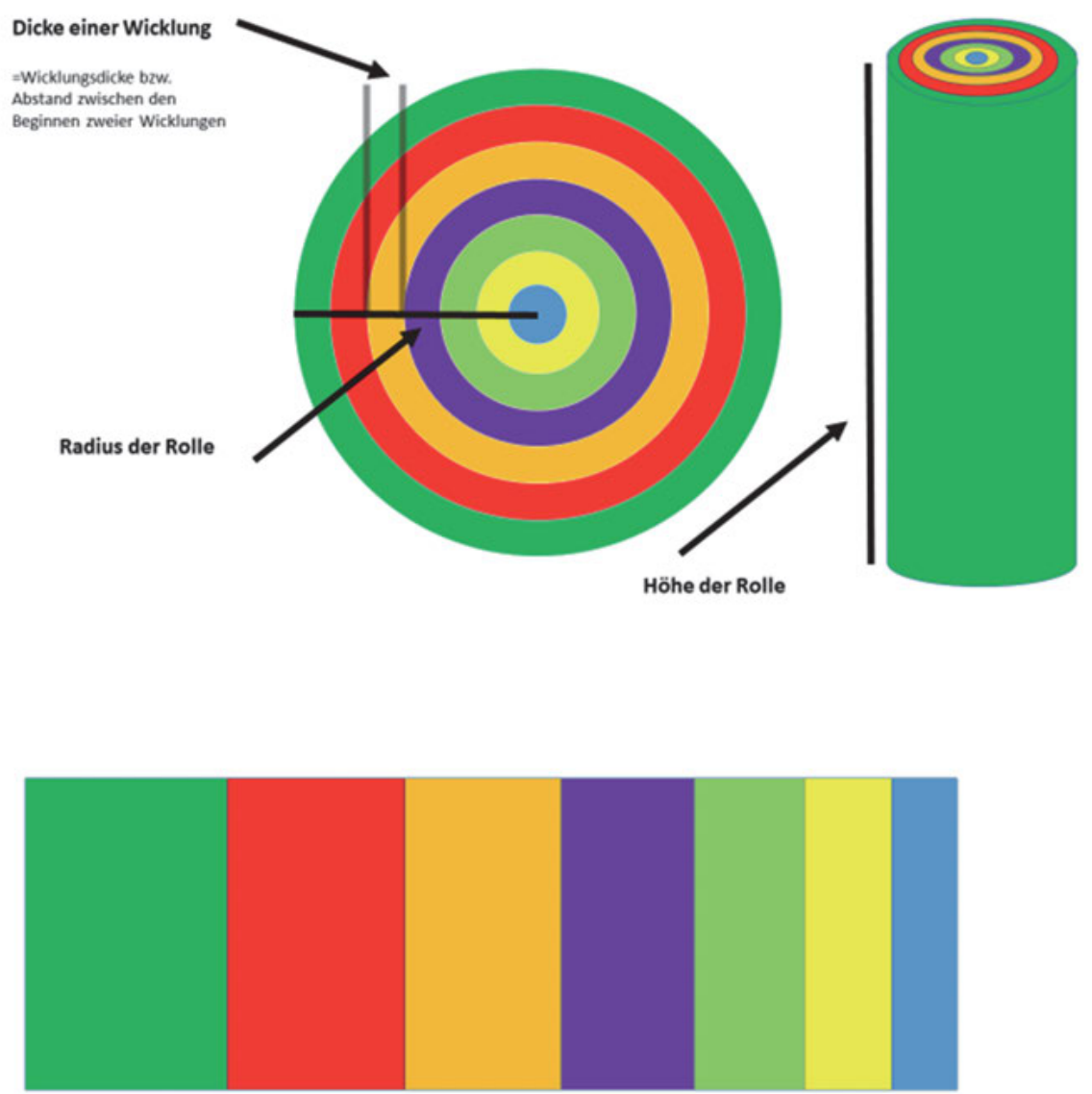

Aufgewickelte Rolle-

Die Wicklungsbreite (=Umfang der einzelnen Kreise) nimmt von links nach rechts (=von außen nach innen) konstant ab (um $2 \pi *$ Wicklungsdicke)

Abb. 46-47: Geschlossene Rolle im Längs-und Querschnitt sowie aufgewickelte Rolle

Der Umfang jedes dieser Kreise (Wicklungen) lässt sich einfach durch die aus der Schule bekannte Formel $2^{\star} \pi^{\star}$ r (äquivalent $\mathrm{zu} \mathrm{d}^{\star} \pi$ ) berechnen, wobei $\mathrm{r}$ für den Radius und d für den Durchmesser steht. Um die Mantelfläche der Rolle über jedem Kreis zu ermitteln, multipliziert man nun einfach mit der Höhe, da die Rollen (approximativ) als Zylinder angesehen werden können. Addiert man nun die Umfänge aller Kreise (Wicklungen) erhält man die Länge der Rolle; addiert man die Mantelflächen aller Zylinder erhält man folglich den Flächen- 
inhalt der Rolle. ${ }^{149}$ Für unsere Zwecke ist wichtig, dass man für die Länge der Rolle auch den Durchschnittsradius $\left(=r^{\star} \pi\right)$ bzw. die Durchschnittsmantelfläche $\left(=r^{\star} \pi^{\star} h\right)$ mit der Anzahl der Wicklungen (der Kreise) multiplizieren kann.

Nun finden sich in der Datenbank chartes (siehe 13.2.2) für etwa 600 Rollen bzw. Rollenteile unter der Angabe „non svolti“ (nicht aufgewickelt) sowohl die Höhenmaße als auch die Durchmesser, ${ }^{150}$ was ausreichend ist, um die (hypothetische) Länge der Rolle im aufgewickelten Zustand bzw. ihren Flächeninhalt bei gegebener Abnahme der Wicklungsbreiten im aufgerollten Zustand (also implizit der Dicke einer Wicklung) zu bestimmen. Die Dicke einer Wicklung bzw. der Abstand zwischen den Wicklungen ist bei allen (geschlossenen) Rollen der Sammlung ähnlich.

\begin{tabular}{|c|c|c|c|c|c|c|}
\hline \multirow{2}{*}{$\begin{array}{l}\text { PHerc.- } \\
\text { Nummer } \\
\text { (ungeöffnete } \\
\text { Rollen) }\end{array}$} & \multirow{2}{*}{$\begin{array}{r}\text { Höhe } \\
h \\
(\mathrm{~cm})\end{array}$} & \multirow{2}{*}{$\begin{array}{l}\text { Durch- } \\
\text { messer } \\
\text { d (cm) }\end{array}$} & \multirow{2}{*}{$\begin{array}{l}\text { Radius } \\
\text { r (cm) }\end{array}$} & \multicolumn{3}{|c|}{$\begin{array}{l}\text { Anzahl (Z) der Wicklungen } \\
\text { bei Änderung der Wicklungsbreite um }\end{array}$} \\
\hline & & & & $0,10 \mathrm{~cm}$ & $0,15 \mathrm{~cm}$ & $0,20 \mathrm{~cm}$ \\
\hline 7 & 9,5 & 5,7 & 2,85 & 179 & 119 & 89 \\
\hline 8 & 9,3 & 4,5 & 2,25 & 141 & 94 & 71 \\
\hline 12 & 8,2 & 4,8 & 2,40 & 151 & 100 & 75 \\
\hline 20 & 10,2 & 5,2 & 2,60 & 163 & 109 & 82 \\
\hline 22 & 15,5 & 4,9 & 2,45 & 154 & 103 & 77 \\
\hline 23 & 10,4 & 6,8 & 3,40 & 214 & 142 & 107 \\
\hline$\ldots$ & & & & & & \\
\hline 1810 & 11,0 & 2,8 & 1,40 & 88 & 59 & 44 \\
\hline
\end{tabular}

Die Anzahl der Wicklungen wurde auf ganze Zahlen gerundet. Sie ergibt sich mittels Division des Radius durch die (konstante) Dicke einer Wicklung im unaufgerollten Zustand (drei verschiedene Werte in der Tabelle). ${ }^{151}$

149 Alternativ kann man die Höhe der Rolle mit den aufaddierten Umfängen der Kreise multiplizieren.

150 Für Hilfe beim Erstellen der Roh-Excel-Tabelle im Rahmen des von mir gegebenen Kurses „Vom Vesuv verschüttet - Die Papyri von Herkulaneum im Digitalen Zeitalter (Eine Einführung)“ im WS 2018/2019 an der Universität Würzburg danke ich Linnea Behncke und Sebastian Schmidt. Rollen, für die keine oder keine vollständigen Angaben vorlagen, wurden nicht berücksichtigt.

151 Die Dicke ergibt sich durch Division der Änderung der Wicklungsbreite im aufgerollten Zustand durch $2 \pi$, ergo entspricht $0,10 \mathrm{~cm}(0,15 \mathrm{~cm} / 0,20 \mathrm{~cm})$ Änderung der Wicklungsbreite etwa einer Dicke von 0,16 $\mathrm{mm}$ (0,24 mm bzw. 0,32 mm). 


\begin{tabular}{rrrr}
\hline $\begin{array}{l}\text { PHerc.-Nummer } \\
\text { (ungeöffnete } \\
\text { Rollen) }\end{array}$ & \multicolumn{2}{c}{ Flächeninhalt (F) der Rolle bei Änderung der Wicklungsbreite um } \\
\hline 7 & $\mathbf{0 , 1 0 ~ c m}$ & $\mathbf{0 , 1 5} \mathbf{~ c m}$ & $\mathbf{0 , 2 0 ~ c m}$ \\
\hline 8 & $15218 \mathrm{~cm}^{2}$ & $10117 \mathrm{~cm}^{2}$ & $7566 \mathrm{~cm}^{2}$ \\
12 & $9264 \mathrm{~cm}^{2}$ & $6176 \mathrm{~cm}^{2}$ & $4665 \mathrm{~cm}^{2}$ \\
20 & $9331 \mathrm{~cm}^{2}$ & $6180 \mathrm{~cm}^{2}$ & $4635 \mathrm{~cm}^{2}$ \\
22 & $13573 \mathrm{~cm}^{2}$ & $9077 \mathrm{~cm}^{2}$ & $6828 \mathrm{~cm}^{2}$ \\
23 & $18363 \mathrm{~cm}^{2}$ & $12282 \mathrm{~cm}^{2}$ & $9182 \mathrm{~cm}^{2}$ \\
$\ldots$ & $23761 \mathrm{~cm}^{2}$ & $15766 \mathrm{~cm}^{2}$ & $11880 \mathrm{~cm}^{2}$ \\
1810 & & & \\
& $4255 \mathrm{~cm}^{2}$ & $2853 \mathrm{~cm}^{2}$ & \\
& & & \\
Summe aller & $8.356 .499 \mathrm{~cm}^{2}$ & $5.573 .886 \mathrm{~cm}^{2}$ & \\
Rollen & & & $4.179 .041 \mathrm{~cm}^{2}$ \\
\hline
\end{tabular}

Um den Flächeninhalt der Rollen zu berechnen, multipliziert man die Mantelfläche des Durchschnittszylinders $\left(2^{\star} \pi^{\star}(\mathrm{r} / 2)^{\star} \mathrm{Z}^{\star} \mathrm{h}\right)$ mit der Anzahl der Wicklungen. Für PHerc. 7 würde man bei Annahme von Änderung der Wicklungsbreite von $1 \mathrm{~mm}$ pro Wicklung $(=0,16 \mathrm{~mm}$ Dicke der Wicklung) also wie folgt rechnen: $2^{\star} \pi^{\star}(2,85 / 2)^{\star} 179^{\star} 9,5=15218 \mathrm{~cm}^{2} .{ }^{152}$

Jedoch haben die hunderten verbogenen Rollen(teile) oft keine echte Zylinderform, ein Umstand dem durch einen Korrekturfaktor unbedingt Rechnung getragen werden muss. Der in der Datenbank angegebene „Durchmesser“ meint in der Regel die breiteste Ausdehnung der Grundfläche der Rolle. Die Grundfläche vieler Rollen ist aber ellipsenförmig; andere gleichen einem polygonen Stumpf. Der Flächeninhalt der Grundfläche ist folglich meist kleiner als der eines Kreises mit entsprechendem Durchmesser. Weitaus gewichtiger ist aber, dass die Rollen in der Höhe auch öfters Kegeln und keinen perfekten Zylindern gleichen. Ferner sind sie teils zu konturlosen und komplexen Gebilden verformt, die eine geringere Mantelfläche als ein Zylinder haben. ${ }^{153}$

Ein Kegel hätte die Mantelfläche $\pi^{\star} \mathrm{r}^{\star} \sqrt{h^{2}+\mathrm{r}^{2}}$, was bei unseren Rollen immer (beträchtlich) kleiner als die Mantelfläche eines Zylinders ist $\left(2^{\star} \pi^{\star} \mathrm{r}^{\star} \mathrm{h}\right)$, mit Grenzwerteigenschaft dahingehend, dass die Mantelfläche des Zylinders bei

152 Für $\pi$ wurde mit 3,14 gerechnet. Die Zahl „zwei“ kürzt sich angenehm heraus.

153 Bezüglich der Rollen sind hier die vergleichsweise wenigen Fälle ausgeklammert, in welchen durch die Krümmung der Rolle die in der Vertikalen gemessene Höhe das Volumen und Mantelfläche der Rolle unterschätzt. 
gleichem Durchmesser fast doppelt so groß ist wie die des Kegels. ${ }^{154}$ Man könnte nun alle erhaltenen Rollen fiktiv als Kegel ansehen, was bedeuten würde, dass die Werte in der Tabelle etwa zu halbieren wären (Multiplikation mit 0,5), um den wahren Flächeninhalt der Rolle zu ermitteln. Man könnte einwenden, dass eine solche Kegelbetrachtung für die erhaltenen Rollenteile etwas übertrieben ist, wogegen man wiederum einwenden könnte, dass die Grundfläche vieler Rollen eher elliptisch ist, was die übertriebene Kegelbetrachtung „ausgleichen“ würde. Die vielen verschiedenen Rollen- bzw. Stumpfformen, welche sich unter den etwa 600 berücksichtigten Nummern verbergen, sind schwer auf einen gemeinsamen Nenner zu bringen. Daher seien in folgender Tabelle exemplarisch drei Korrekturfaktoren genutzt, wobei der Faktor 0,5 - der in Richtung Kegel bzw. in Richtung von dessen Mantelfläche geht - am stärksten korrigiert. ${ }^{155}$

\begin{tabular}{|c|c|c|c|c|c|c|c|c|c|}
\hline \multirow[t]{2}{*}{$\begin{array}{l}\text { PHerc.- } \\
\text { Nummer } \\
\text { (ungeöffnete } \\
\text { Rollen) }\end{array}$} & \multicolumn{3}{|c|}{$\begin{array}{l}\text { Flächeninhalt (F) der Rolle } \\
\text { bei Änderung der Wick- } \\
\text { lungsbreite } \\
\text { um } 0,10 \mathrm{~cm} \\
\text { Korrekturfaktor }\end{array}$} & \multicolumn{3}{|c|}{$\begin{array}{l}\text { Flächeninhalt (F) der } \\
\text { Rolle bei Änderung der } \\
\text { Wicklungsbreite } \\
\text { um } 0,15 \mathrm{~cm} \\
\text { Korrekturfaktor }\end{array}$} & \multicolumn{3}{|c|}{$\begin{array}{l}\text { Flächeninhalt (F) der } \\
\text { Rolle bei Änderung der } \\
\text { Wicklungsbreite } \\
\text { um } 0,20 \mathrm{~cm} \\
\text { Korrekturfaktor }\end{array}$} \\
\hline & 0,75 & 0,6 & 0,5 & 0,75 & 0,6 & 0,5 & 0,75 & 0,6 & 0,5 \\
\hline 7 & 11413 & 9131 & 7609 & 7588 & 6070 & 5058 & 5675 & 4540 & 3783 \\
\hline 8 & 6948 & 5559 & 4632 & 4632 & 3706 & 3088 & 3499 & 2799 & 2333 \\
\hline 12 & 6998 & 5599 & 4666 & 4635 & 3708 & 3090 & 3476 & 2781 & 2317 \\
\hline 20 & 10180 & 8144 & 6787 & 6808 & 5446 & 4538 & 5121 & 4097 & 3414 \\
\hline 22 & 13772 & 11018 & 9182 & 9211 & 7369 & 6141 & 6886 & 5509 & 4591 \\
\hline $\begin{array}{c}23 \\
\ldots\end{array}$ & 17820 & 14256 & 11880 & 11825 & 9460 & 7883 & 8910 & 7128 & 5940 \\
\hline 1810 & 3191 & 2553 & 2128 & 2140 & 1712 & 1427 & 1596 & 1277 & 1064 \\
\hline $\begin{array}{r}\text { Alle Rollen } \\
\text { (in Mio. } \mathrm{cm}^{2} \text { ) }\end{array}$ & 6,3 & 5,0 & 4,2 & 4,2 & 3,3 & 2,8 & 3,1 & 2,5 & 2,1 \\
\hline
\end{tabular}

154 Der Ausdruck $\pi^{\star} r^{\star} \sqrt{h^{2}+r^{2}}$ tendiert für die inneren konzentrischen Kreise immer mehr gegen $\pi^{\star} r^{\star} h$. Selbst für den größten Außenkreis ist bei der weit überwiegenden Zahl der Tabellenwerte der Ausdruck recht nahe an $\pi^{\star} \mathrm{r}^{\star} \mathrm{h}$.

155 Gewiss gibt es auch vereinzelte Rollen, die etwa nur an einem Ende nach oben ragen, und deren Mantelfläche somit selbst durch die Mantelfläche eines Kegels noch überschätzt wäre. 
Nun hat die durchschnittliche Herkulanische Papyrusrolle etwa eine Höhe von $22 \mathrm{~cm} .{ }^{156}$ Damit läge die fiktive Länge der noch $\mathrm{zu}$ entrollenden (fiktiven vollständigen) Rollen der Sammlung je nach Wicklungsbreite und Korrekturfaktor zwischen 950 und 2850 Metern. Bei Annahme von 6,5 cm Abstand zwischen den Kolumnenbeginnen (interkolumnarer Raum) kommt man ja nach Annahmen auf zwischen 15.000 und 43.000 (vollständige!) Kolumnen. ${ }^{157}$

\begin{tabular}{|c|c|c|c|c|c|c|c|c|c|}
\hline & \multicolumn{9}{|c|}{$\begin{array}{l}\text { Text in allen noch unaufgerollten Rollen } \\
\text { Bei Änderung der Wicklungsbreite um }\end{array}$} \\
\hline & \multicolumn{2}{|c|}{$\begin{array}{l}0,10 \mathrm{~cm} \\
\text { Korrekturfaktor }\end{array}$} & \multicolumn{3}{|c|}{$\begin{array}{l}0,15 \mathrm{~cm} \\
\text { Korrekturfaktor }\end{array}$} & \multicolumn{3}{|c|}{$\begin{array}{l}0,20 \mathrm{~cm} \\
\text { Korrekturfaktor }\end{array}$} & \\
\hline & 0,75 & 0,6 & 0,5 & 0,75 & 0,6 & 0,5 & 0,75 & 0,6 & 0,5 \\
\hline Länge (m) & 2849 & 2279 & 1892 & 1900 & 1520 & 1267 & 1425 & 1140 & 950 \\
\hline Kolumnen (Taus.) & 43 & 35 & 29 & 29 & 23 & 19 & 22 & 18 & 15 \\
\hline Wörter (Mio.) & 4,9 & 3,9 & 3,3 & 3,3 & 2,6 & 2,2 & 2,5 & 2,0 & 1,6 \\
\hline OCT-Seiten (Taus.) & 25 & 20 & 16 & 16 & 12 & 11 & 12 & 10 & 8 \\
\hline OCT-Bände & 98 & 79 & 65 & 65 & 52 & 44 & 49 & 39 & 33 \\
\hline
\end{tabular}

Im Schnitt sind etwa $17 \mathrm{~cm}$ dieser Höhe beschrieben (etwas mehr als $2 \mathrm{~cm}$ oberer und unterer Rand), d.h. die Höhe einer Kolumne entspricht etwa $17 \mathrm{~cm}$, in welcher durchschnittlich ungefähr 32 Zeilen Platz finden. ${ }^{158}$ Bei Annahme von etwa 3,5 Wörtern pro Zeile ergibt sich, ${ }^{159}$ dass in den heute noch unaufgerollten Rollen der Herkulanischen Sammlung in jedem Fall über 1,5 Mio. Wörter zu lesen wären. Nun habe ich für eine typische Seite eines Prosatextes in der Reihe Oxford Classical Texts (OCT) etwa 200 Wörter und für einen typischen OCTProsaband etwa 250 Seiten angenommen. ${ }^{160}$ Je nach Annahme der beiden Vari-

156 Vgl. Cavallo, Libri scritture scribe (wie Anm. 65), 16. Für die folgenden Berechnungen wurde die plausible Annahme getroffen, dass sich die Rollenteile letztlich hinsichtlich der Ränder bzw. keinen Rändern ergänzen, was auch durch das Faktum, dass viele der Rollenteile ganz offenbar von identischen Rollen stammen, die in Stücke gebrochen sind, gerechtfertigt ist.

157 Für die Breite vgl. Cavallo, Libri scritture scribe (wie Anm. 65), 18-19 (die Zahl ist im Hinblick auf die Kolumnenzahl konservativ gewählt). Zum Vergleich: Philodems De morte IV, von dem uns nur die letzten ca. 40 Kolumnen unvollständig erhalten sind, hatte 118 Kolumnen.

158 Cavallo, Libri scritture scribe (wie Anm. 65), 18.

159 Schätzung anhand der Durchschnittszeilenlänge von etwa 18 Buchstaben pro Zeile, vgl. Cavallo, Libri scritture scribe (wie Anm. 65), 18.

160 Eigene Zählung bzw. eigene Schätzung. Die Werte sind eher konservativ angesetzt, d.h. tendenziell wurden eher mehr Wörter pro Seite und mehr Seiten pro Band geschätzt. Man 
ablen Änderung der Wicklungsbreite (bzw. Dicke der Wicklung) und Korrekturfaktor wäre somit noch Text im Umfang von etwa 8000 bis 25.000 OCT-Seiten (entspricht etwa 30-100 Bänden) in den Rollen verborgen. Diese würden wohl von etwa 300 verschiedenen (fiktiv vollständigen) Rollen (Werken) stammen (300 mit erheblicher Varianz), denen die 600 für die Tabellen-Berechnungen erfassten Rollenteile (und zusätzlich dutzende nicht erfasste Rollenteile) zuzurechnen sind. In einer „Kontrollrechnung“ ergäben ca. 3-10 fiktiv-vollständige Rollen einen OCT-Band, was plausibel ist (je nach durchschnittlicher Rollenlänge).

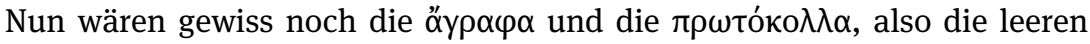
Blätter zu Beginn und Ende von Rollen, sowie teils umbillici ${ }^{161}$ in Abzug zu bringen (welche vielleicht durch mehrere ob fehlender Angaben in meiner Rechnung nicht berücksichtigter Rollen ausgeglichen würden), und einige würden vielleicht mit noch größerer (übertriebener) Vorsicht obige Variablen anders (noch konservativer) ansetzen, so dass ich mich vorsichtshalber in meiner Bewertung nur auf die niedrigsten Werte in der Tabelle (ganz rechts - Korrekturfaktor 0,5 und Wicklungsbreitenänderung $0,2 \mathrm{~cm}$ ) beschränken will. Selbst in diesem nach meinen Berechnungen „schlechtestem“ Fall wären durch das virtual unrolling noch ca. 8000 „Referenzseiten“ antiken Textes zu gewinnen. Da bisher eher die besser erhaltenen Rollen geöffnet wurden und die lateinischen Rollen ganz offenbar eher zu den schlechter zu öffnenden Rollen gehörten, dürfte das bisherige Übergewicht griechischer zu lateinischen Papyri von etwa 10:1 bei den ungeöffneten Rollen weniger ausgeprägt sein. Unter 8000 neuen Seiten wären gewiss etliche hunderte Seiten lateinischer Literatur. ${ }^{162}$ Bei den grob geschätzt 300-400 Werken in den noch unaufgerollten Rollen wären in den Fällen, wo heute fast vollständige Rollen erhalten sind, wohl $70-100 \%$ des Textes wiederherstellbar oder kombinierbar, bei den kleineren „Stumpfresten“ teils weniger als $10 \%$.

Die Zahlen zeigen, welch große Menge an Text aus den Rollen theoretisch noch zu gewinnen ist. Allerdings beruhen die Überlegungen und Rechnungen auf der Voraussetzung, dass das virtual unrolling relativ maschinell-problemlos funktioniert und insbesondere im Inneren der Rollen keine Fusionen (Verschmelzungen) von Wicklungen stattgefunden haben, welche den Text inner-

erkennt in der Tabelle auch, dass die Werte für $0,10 \mathrm{~cm} / 0,5$ Korrektur sowie $0,15 \mathrm{~cm} / 0,75$ Korrektur bis auf Rundungsfehler identisch sind.

161 Einige Rollen wurden um dünne Stangen (umbillici) gewickelt, welche am Ende der Rollen angebracht waren.

162 Jedoch gilt es für diese Überlegungen Unterschiede im Format zu berücksichtigen. 
lich zerstört und weitestgehend unlesbar gemacht haben. Die Identifikation der Wicklungen über beinahe die ganze Höhe geschlossener Rollen (siehe 11.2) und die bisherigen virtual unrolling-Versuche (11.3) sprechen erfreulicherweise tendenziell gegen solche inneren, vernichtenden Verschmelzungen in größerem Umfang (bei mehreren Rollen). Jedenfalls sollte durch diese erste, mathematisch-bibliometrische untermauerte Quantifizierung des textuellen Potentials noch geschlossener Rollen(teile) deutlich geworden sein, welche Menge an neuem Text bei einem Erfolgt des virtual unrolling im Raum steht und was unter „Da ist noch viel Text in den ungeöffneten Rollen!“ konkret-quantitativ zu verstehen ist. Selbst bei den (realistisch gewählten) konservativsten Variablen kommt man auf über 8000 OCT-Seiten, d.h. über 30 Bände neuen Text. Auch wenn wir hier nochmals etwaige Probleme in Abzug bringen, dürften doch mindestens über ein Dutzend moderne Norm-Bände antiker Literatur zu gewinnen sein. Die neuen Texte würden ein breites Spektrum von Autoren und Gattungen abdecken, gewiss mit Dominanz griechisch-epikureischer Texte. 